EPJ Web of Conferences 40, 16006 (2013)

DOI: $10.1051 /$ epjconf/20134016006

(C) Owned by the authors, published by EDP Sciences, 2013

\title{
Holographic images reconstructed from GMR-based fringe pattern
}

\author{
Daisuke Kato ${ }^{1}$, Kenichi Aoshima ${ }^{1}$, Kenji Machida $^{1}$, Akira Emoto ${ }^{2}$, Hidekazu Kinjo ${ }^{1}$, Kiyoshi Kuga ${ }^{1}$, Hiroshi Ono ${ }^{3}$ \\ Takayuki Ishibashi ${ }^{4}$, Hiroshi Kikuchi ${ }^{1}$, Naoki Shimidzu $^{1}$ \\ ${ }^{1}$ NHK Science \& Technology Research Laboratories, 1-10-11 Kinuta, Setagaya-ku, Tokyo 157-8510, Japan \\ ${ }^{2}$ National Institute of Advanced Industrial and Science Technology, Tsukuba, Ibaraki 305-8561, Japan \\ ${ }^{3}$ Department of Electrical Engineering, Nagaoka University of Technology, 940-2188, Japan \\ ${ }^{4}$ Department of Materials Sciences and Technology, Nagaoka University of Technology, 940-2188, Japan
}

\begin{abstract}
We have developed a magneto-optical spatial light modulator (MOSLM) using giant magneto-resistance (GMR) structures for realizing a holographic three-dimensional (3D) display. For practical applications, reconstructed image of hologram consisting of GMR structures should be investigated in order to study the feasibility of the MOSLM. In this study, we fabricated a hologram with GMR based fringe-pattern and demonstrated a reconstructed image. A fringe-pattern convolving a crossshaped image was calculated by a conventional binary computer generated hologram (CGH) technique. The CGH-pattern has 2,048 $\times 2,048$ with $5 \mu \mathrm{m}$ pixel pitch. The GMR stack consists of a Tb-Fe-Co/CoFe pinned layer, a Ag spacer, a Gd-Fe free layer for light modulation, and a Ru capping layer, was deposited by dc-magnetron sputtering. The GMR hologram was formed using photo-lithography and Krion milling processes, followed by the deposition of a Tb-Fe-Co reference layer with large coercivity and the same Kerr-rotation angle compared to the free layer, and a lift-off process. The reconstructed image of the ON-state was clearly observed and successfully distinguished from the OFF-state by switching the magnetization direction of the free-layer with an external magnetic field. These results indicate the possibility of realizing a holographic 3D display by the MOSLM using the GMR structures.
\end{abstract}

\section{Introduction}

Holography $[1,2]$ has emerged as a very attractive method for 3D imaging because it reconstructs the same wavefronts of light that are emitted from objects. The study of electronic holography has been actively carried out using various electronically addressable spatial light modulator (SLM) technologies, such as magneto-optical (MO) SLM [3] and liquid crystal SLM [4-5]. Especially, the MOSLM has advantages of high speed switching and no volatility. The MOSLM driven by an external magnetic field, which consists of garnet film, has a large degree of the light modulation compared to other MOSLMs. However, higher resolution pixels are not easy to achieve because the magnetic field tend to diverge and may switch the unintended magnetic pixels next to the intended pixel [6]. The MOSLM driven by the electrostrictive effect has been developed for lower power consumption, however it may not be able to provide a higher resolution because it needs a complex structure of electrode [7]. Therefore, the viewing-zone angles of reconstructed images using these MOSLMs were very narrow because the pixel pitches of them were not small enough. So, we have studied an ultra-fine SLM which may have potential to display 3D images with a wide viewing-zone angle, and proposed a MOSLM using giant magneto-resistance (GMR) structures which is driven by spin-transfer-switching (STS) [8], which we call SpinSLM. Small pixel size with narrow pixel pitch of the Spin-SLM can be fabricated since the magnetization direction of pixel is controlled by an electric current flowing through an intended pixel with no crosstalk on the adjacent pixels. The Spin-SLM has potential to be the 3D display with high frame-rate, because the operation speed of the STS is as fast as tens of nanoseconds or less. Previously, we have fabricated a light modulation device with a single submicron magnetic pixel and successfully obtained MO image of the single submicron magnetic pixel driven by STS [9].

Since the degree of the light modulation of the magnetic materials used in the Spin-SLM is very small, reconstructed images from the Spin-SLM could be very limited or may not be visible. Therefore, in order to study the feasibility of the Spin-SLM, a reconstructed image from the Spin-SLM should be investigated. In this study, instead of fabricating large scale Spin-SLM in order to study reconstructed images from them, we take relatively simple and easy way to fabricate hologram

This is an Open Access article distributed under the terms of the Creative Commons Attribution License 2.0, which permits unrestricted use, distribution, and reproduction in any medium, provided the original work is properly cited. 


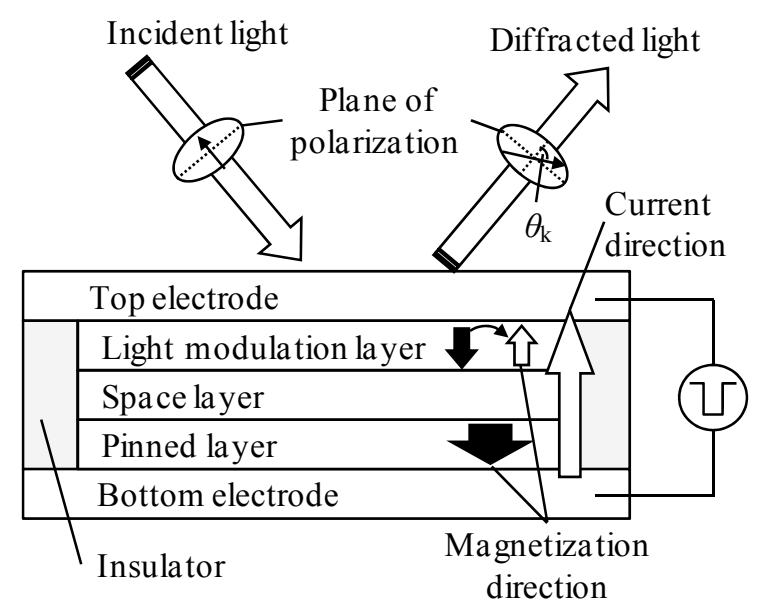

Fig.1 Illustrations of the Spin-SLM and the MO Kerr effect

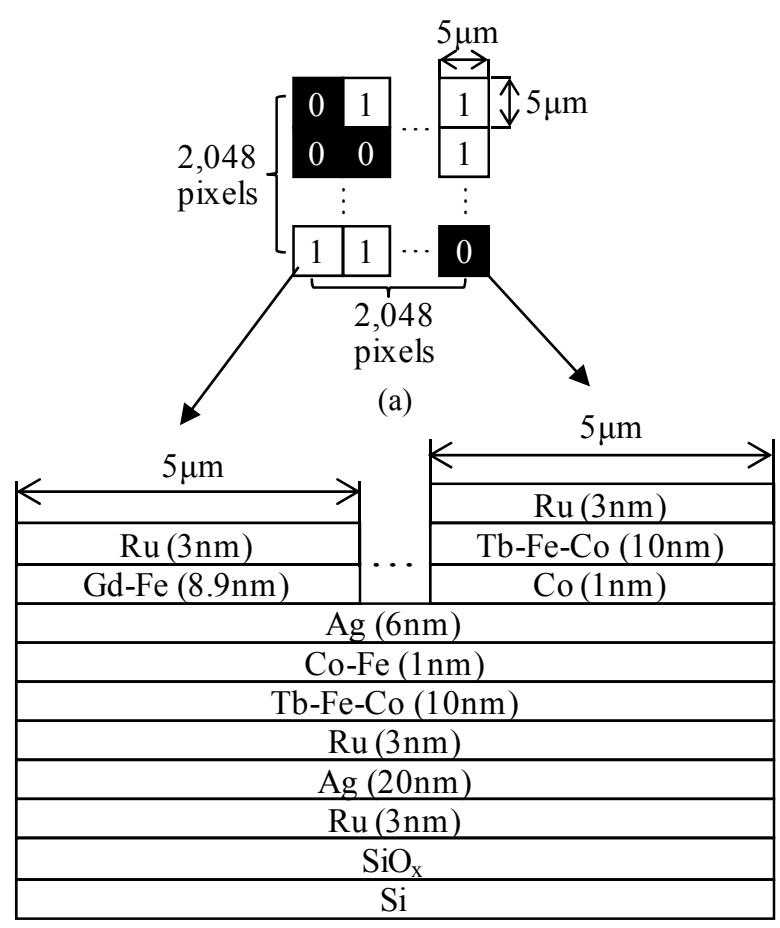

(b)

Fig.2 Structure of the fabricated GMR hologram :

(a) Binary hologram, (b) Fabricated GMR hologram

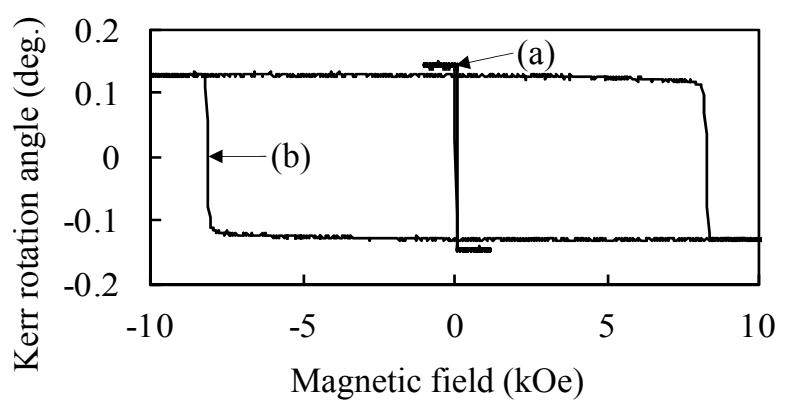

Fig.3 Characteristics of Kerr rotation angle for each MO layer : (a) Gd-Fe light modulation layer,

(b) $\mathrm{Tb}-\mathrm{Fe}-\mathrm{Co}$ reference layer

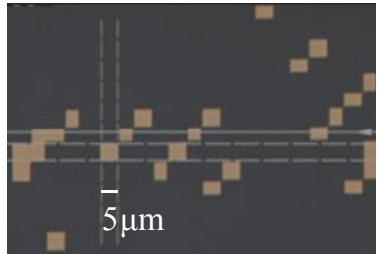

(a)

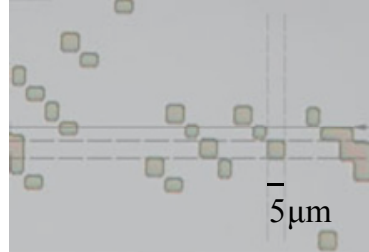

(b)
Fig.4 Optical micrographs :

(a) Photo mask, (b) Photo resist pattern

patterns made of GMR multilayers (GMR hologram) without electrode for driving which can be fabricated with relatively simple lithography technique, and evaluated the capability of displaying images with magnetic materials.

\section{Spin-SLM}

Figure 1 shows a cross-sectional schematic illustration of the Spin-SLM. Light modulation pixel of the Spin-SLM is sandwiched by the bottom electrode made of cupper and the top transparent electrode made of In-Zn-O. Each light modulation pixel is comprised of a light modulation magnetic layer, a space layer, and a magnetically pinned layer. This structure is commonly called a Current-Perpendicular-to-Plane (CPP-) GMR structure. Here, the pinned layer has large coercivity while the light modulation layer has small coercivity.

The Spin-SLM is driven by a pulse current flowing from the top to the bottom (STS), which reverse the magnetization direction of the light modulation layer of the Spin-SLM. The pinned layer has the function of discriminating the spin-direction of the conduction electrons. Figure 1 shows that the magnetization direction of the light modulation layer and that of the pinned layer are parallel. In this case, when the pulse current is flowing from the bottom to the top electrode, only spindown electrons from the top electrode can pass through the pinned layer and spin-up electrons induce the magnetization direction of the light modulation layer to be switched to anti parallel direction to that of the pinned layer by the spin transfer effect. Contrary, when the pulse current is flowing to the opposite direction, the magnetization direction of the light modulation layer is switched to parallel direction to that of the pinned layer. Thus, the magnetization direction of the light modulation layer is controlled by the direction of pulse current. When polarized incident light is introduced to the light modulation layer, the plane of polarization of the diffracted light in the light modulation layer rotate the Kerr rotation angle of $+\theta_{\mathrm{k}}$ or $-\theta_{\mathrm{k}}$, which depends on the magnetization direction of the light modulation layer (figure 1). By placing an appropriately oriented analyzer at the output of the system (not shown), each light modulation pixel can be black or white depending on the magnetization direction of the light modulation layer and the analyzer orientation. Therefore, fringe-pattern for 3D images is produced by the Spin-SLM and the analyzer. 


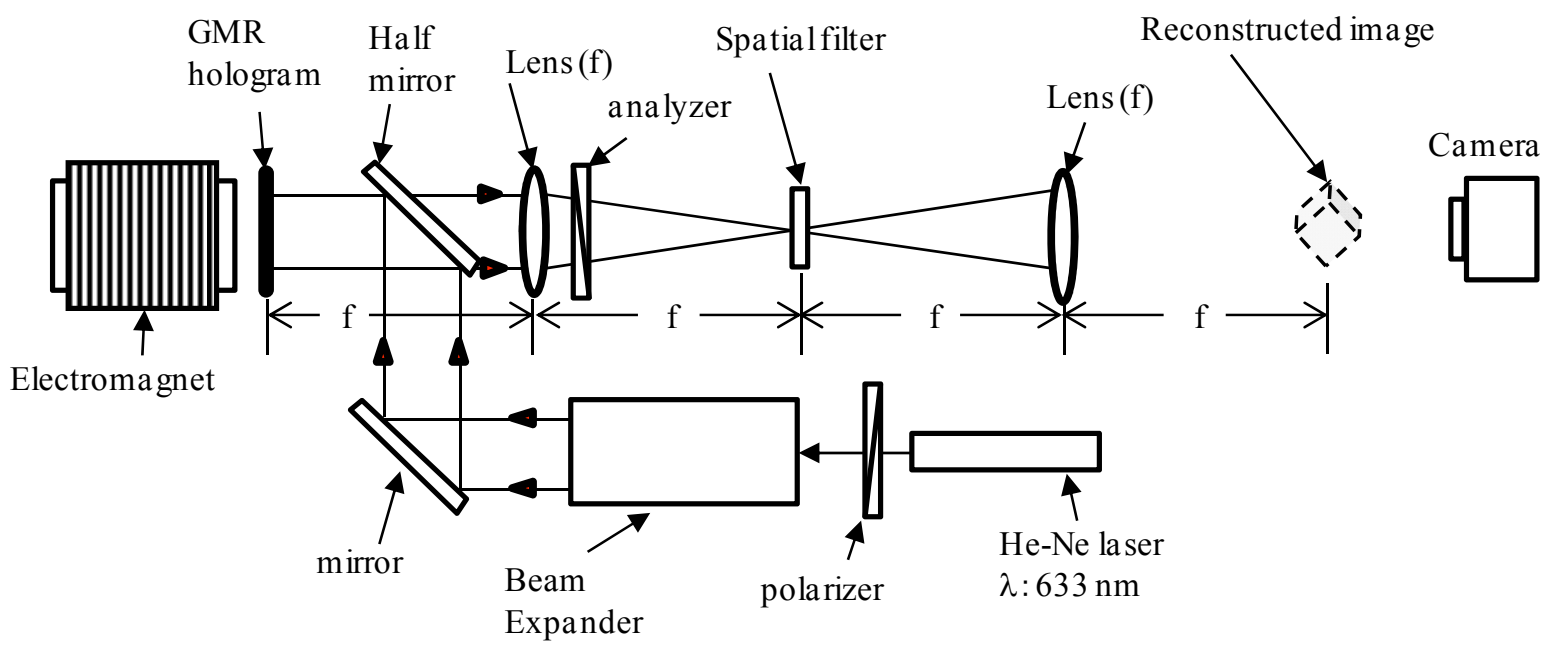

Fig.5 Optical configuration

\section{Results}

\subsection{Fabrication of the GMR hologram}

A fringe-pattern convolving a cross-shaped image was calculated using a conventional computer generated hologram $(\mathrm{CGH})$ technique. The calculated CGH-pattern has $2,048 \times 2,048$ array layout with $5 \mu \mathrm{m}$ pixel pitch.

The GMR stacks of the GMR hologram, which are comprised of Tb-Fe-Co $(10 \mathrm{~nm}) / \mathrm{Co}-\mathrm{Fe}(1 \mathrm{~nm})$ pinned layer, a $\mathrm{Ag}(6 \mathrm{~nm})$ space layer, a Gd-Fe $(8.9 \mathrm{~nm})$ light modulation layer and a $\mathrm{Ru}(3 \mathrm{~nm})$ capping layer, were deposited with a DC magnetron sputtering system at a base pressure of less than $2 \times 10^{-7} \mathrm{~Pa}$. The GMR hologram was patterned using photo lithography and $\mathrm{Kr}$ ion milling process. The milling was stopped just before the Ag space layer followed by a deposition of reference layers comprised of Co $(1 \mathrm{~nm}) / \mathrm{Tb}-\mathrm{Fe}-\mathrm{Co}(10 \mathrm{~nm})$ magnetic layers and a $\mathrm{Ru}(3 \mathrm{~nm})$ capping layer using an ion beam sputtering system. Finally, the GMR hologram is completed after a lift-off process.

Figure 2(a) shows an illustration of a binary hologram and figure 2(b) shows a cross-sectional schematic illustration of the fabricated GMR hologram. In figure 2, the pixels referred to " 1 " are comprised of the $\mathrm{Tb}-\mathrm{Fe}-\mathrm{Co} / \mathrm{Co}-\mathrm{Fe}$ pinned layer, the $\mathrm{Ag}$ space layer and Gd-Fe light modulation layer, we call it Gd-Fe light modulation pixels. The pixels referred to " 0 " are comprised of the $\mathrm{Tb}-\mathrm{Fe}-\mathrm{Co} / \mathrm{Co}-\mathrm{Fe}$ pinned layer, the $\mathrm{Ag}$ space layer and the $\mathrm{Co} / \mathrm{Tb}-\mathrm{Fe}-\mathrm{Co}$ magnetic layers, we call it $\mathrm{Tb}-\mathrm{Fe}-\mathrm{Co}$ reference pixels.

Here, a Kerr rotation angle of the Gd-Fe light modulation layer was about 0.14 degrees at wavelength of $780 \mathrm{~nm}$ as shown in figure 3(a). The Tb-Fe-Co reference layer had a large coercivity and was controlled to have same Kerr-rotation angle compared to the Gd-Fe light modulation layer as shown in figure 3(b),

Figure 4(a) shows an optical micrograph of a part of the photo mask, and figure 4(b) shows an optical micrograph of the photo resist pattern. As you can see in the figure 4 , patterns of the photo mask and the photo resist are identical including minimum patterns of $5 \mu \mathrm{m}$ dots, which indicate that precise patterning was carried out as small as $5 \mu \mathrm{m}$.

\subsection{Optical configuration}

Figure 5 shows a schematic illustration of the optical setup to demonstrate the reconstructed image from the GMR hologram. The incident light source was a He-Ne leaser $(\lambda=632.8 \mathrm{~nm})$. Polarized incident light was expanded and introduced to the GMR hologram through a half mirror. Diffracted light travelled through an analyzer, which was set to cut the diffracted light with a specific plane of polarization. The spatial filter was used to selectively capture the first-order diffracted image with the camera. In addition, an electromagnet was used to apply an external magnetic field to the GMR hologram.

\subsection{Optical observation of the GMR hologram}

The external magnetic field of over $10 \mathrm{kOe}$ was applied to the GMR hologram to initialize the magnetization direction in the $\mathrm{Tb}-\mathrm{Fe}-\mathrm{Co}$ reference pixels.

We observed the cross-shaped image and the image showed an on-state and an off-state by switching the magnetization direction of the Gd-Fe light modulation pixels with the external magnetic field of $\pm 200 \mathrm{Oe}$. Figure 6 show photographs of reconstructed images. Figure 6(a) shows the on-state and figure 6(b) shows the off-state of the reconstructed image. In figure 6 , both the on-state and the off-state are clearly distinguishable in spite of the small Kerr rotation angle of the Gd-Fe light modulation layer, which is about 0.14 degrees (figure 3 ).

We discuss how to obtain the reconstructed images with the on-state and the off-state from the GMR hologram. For the diffracted light in the Gd-Fe light modulation pixel, the plane of polarization of that is rotated to clockwise $\left(\theta_{\mathrm{k}}\right)$ or counter clockwise $\left(-\theta_{\mathrm{k}}\right)$ respectively, according to the magnetization direction of the Gd-Fe light modulation pixel. On the other hand, for the diffracted light in the Tb-Fe-Co reference pixel, the plane of polarization is only rotated to counter clock wise $\left(-\theta_{\mathrm{k}}\right)$ because the magnetization direction of the Tb-Fe- 
Co reference pixels does not change with the external magnetic field of this experiment. Here, the analyzer is set to cut off the maximum amount of diffracted light whose plane of polarization is rotated at $-\theta_{\mathrm{k}}$. Therefore, when the magnetization direction of the Gd-Fe light modulation pixels aligned with that of $\mathrm{Tb}-\mathrm{Fe}-\mathrm{Co}$ reference pixels, the analyzer cut off the maximum amount of diffracted light (the off-state). Contrarily, if the magnetization direction of the Gd-Fe light modulation pixels is switched to the opposite direction by applying the external magnetic field, diffracted light in the Gd-Fe light modulation pixels can pass through the analyzer (the on-state). Through this experiment, we confirmed that the Spin-SLM would be able to display 3D movies if each pixel of the Spin-SLM could be driven by STS.

Here, the intensity of the diffracted light was very low at below the noise level of an experimental condition with a detector, although we can see the reconstructed image via a lens. Therefore, it was difficult to measure precise intensity of the diffracted light. This is because the Kerr rotation angle was very small, which was about 0.14 degrees. However, we could roughly estimate the diffraction efficiency of the GMR hologram at $0.01 \%$ or less, which was derived from the ratio of the intensity of the incident light and the intensity of the diffracted light which was almost equal to the noise level.

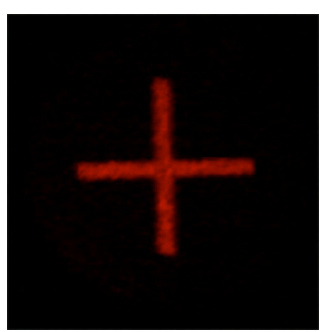

(a)

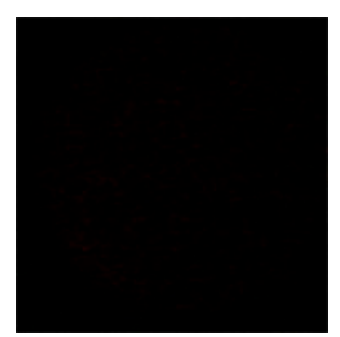

(b)
Fig.6 Reconstructed image (cross-shaped image) :

(a) On-state, (b) Off-state

\section{Conclusions}

A fringe-pattern convolving a cross-shaped image was calculated by a conventional binary CGH technique. The GMR hologram was composed of the Gd-Fe light modulation layer, which is the same magnetic materials used in the Spin-SLM device, and the Tb-Fe-Co reference layer. We successfully observed the reconstructed MO images and the images showed the onstate and the off-state by applying the external magnetic field. As a result, we have demonstrated the potential of the Spin-SLM to realize holographic 3D images.

\section{Acknowledgment}

This research has been partially supported by National Institute of Communications Technology (NICT) in Japan.

\section{References}

1. D. Gabor, Nature, 161, 4098 (1948)

2. E.N. Leith, J. Upatnieks, J. Opt. Soc. Am., 52, 10 (1962)

3. D. Psaltis, E.G. Paek, S.S. Venkatesh, Opt. Eng., 23, 6 (1984)

4. F. Mok, J. Diep, H. Liu, D. Psaltis, Opt. Lett., 11, 11 (1986)

5. T. Mishina, M. Okui, F. Okano, Appl. Opt., 41, 8 (2002)

6. J.A. Davis, J. Gamlieli, G.W. Bach, Appl. Opt., 27, 24 (1988)

7. J.H. Park, K. Nisimura, M. Inoue, D.H. Lee, J.K. Cho, J. Appl. Phys., 91, 10 (2002)

8. K. Aoshima, Y. Hashimoto, N. Funabashi, K. Machida, K. Kuga, H. Kikuchi, N. Shimidzu, T. Ishibashi, J. Appl. Phys., 111, 7 (2012)

9. N. Funabashi, K. Aoshima, K. Machida, K. Kuga, H. Kikuchi, N. Shimidzu, T. Ishibashi, MORIS 2011, p.155 (2011) 